\title{
Belphégor
}

\section{Multiples et multiplications dans Peter Pan}

\section{Monique Chassagnol}

\section{OpenEdition}

\section{Journals}

Édition électronique

URL : http://journals.openedition.org/belphegor/383

DOI : 10.4000/belphegor.383

ISSN : 1499-7185

Éditeur

LPCM

Édition imprimée

Date de publication : 10 janvier 2011

\section{Référence électronique}

Monique Chassagnol, « Multiples et multiplications dans Peter Pan », Belphégor [En ligne], 10-3 | 2011, mis en ligne le 10 janvier 2013, consulté le 20 avril 2019. URL : http://journals.openedition.org/ belphegor/383 ; DOI : 10.4000/belphegor.383

Ce document a été généré automatiquement le 20 avril 2019.

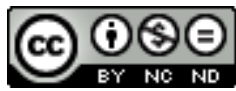

Belphégor est mis à disposition selon les termes de la Licence Creative Commons Attribution - Pas d'Utilisation Commerciale - Pas de Modification 4.0 International. 


\title{
Multiples et multiplications dans Peter Pan
}

\author{
Monique Chassagnol
}

Une nuit de décembre ${ }^{1}$, un vendredi, jour de Vénus, Peter Pan s'introduit une seconde fois chez les Darling à Londres, par la fenêtre de la nursery, pour venir chercher son ombre perdue lors d'une précédente visite. Il surprend d'abord Mrs. Darling puis il réveille la petite Wendy et lui raconte son histoire, en forme d'analepse, en deux épisodes distincts, séparés par plusieurs chapitres: dès sa naissance, horrifié en entendant ses parents envisager son avenir, il s'est envolé par la fenêtre de sa nursery londonienne. À son retour, une autre nuit, il a découvert, derrière la fenêtre désormais fermée et munie de barreaux, un petit garçon, double de lui-même, endormi dans son propre lit, occupant sa place auprès de sa mère. Convaincu que sa mère l'a oublié, il s'est alors envolé vers Neverland, jaloux et furieux, décidé à ne jamais devenir adulte. Wendy, petite fille pas si sage, fascinée par l'histoire de ce jeune rebelle, accepte avec ravissement de le suivre. Et à nouveau Peter s'envole, une nuit, par la fenêtre d'une nursery, accompagné cette fois des trois enfants Darling. Il y reviendra à plusieurs occasions, de la même manière. D'où la multiplication de scènes de départs et de retours nocturnes, d'intrusion et d'exclusion et la récurrence de l'image de la fenêtre ouverte/fermée. On pense au poème de Baudelaire :

Celui qui regarde du dehors à travers une fenêtre ouverte, ne voit jamais autant de choses que celui qui regarde une fenêtre fermée. [...] Ce qu'on peut voir au soleil est toujours moins intéressant que ce qui se passe derrière une vitre. Dans ce trou noir ou lumineux vit la vie, rêve la vie, souffre la vie ${ }^{2}$.

Parmi les aventures sans cesse renouvelées de Peter Pan à mesure de ses nombreux allers et retours du monde réel à son monde imaginaire, le narrateur en sélectionne une seule : celle vécue avec les jeunes Darling. Et de cette aventure il choisit un seul de ses nombreux épisodes. À la fin du chapitre 7, à l'issue d'un jeu sur la prétérition, écartant sept - ou plutôt huit, l'une étant présentée comme double - des nombreuses histoires possibles, il se tourne vers son public, le prend à témoin, le sollicite. De nouveaux récits, très prometteurs, sont alors esquissés, entrepris puis interrompus, aussitôt remplacés par l'ébauche d'un autre, puis d'un autre encore. L'usage de « we » suggère un choix commun, 
une coopération entre le narrateur adulte, - c'est bien lui qui en fin de compte tire au sort - et l'enfant lecteur :

To describe them all would require a book as large as an English-Latin, LatinEnglish Dictionary, and the most we can do is to give one as a specimen of an average hour on the island. The difficulty is which one to choose. Should we take the brush with the redskins at Slightly Gulch? It was a sanguinary affair [...] - but we have not decided yet that this is the adventure we are to narrate; Perhaps a better one would be the night attack by the redskins on the house under the ground [...] Or we might tell how Peter saved Tiger Lily's life [...] Or we could tell of that cake the pirates cooked so that the boys might eat it and perish [...] Or suppose we tell of the birds that were Peter's friends [...] etc.

Which of these adventures shall we choose? The best way will be to toss for it. I have tossed, and the lagoon has won. This almost makes one wish that the gulch or the cake or Tink's leaf had won. Of course I could do it again, and make it best out of the three; however, perhaps fairest to stick to the lagoon ${ }^{3}$.

Ainsi, après bien des hésitations et retours en arrière, peut commencer le chapitre 8 , "The Mermaid Lagoon» et sa suite. Le coup de dé, devenu déterminant, constituant de la création littéraire, rend le récit aléatoire.

Toujours entre diégèse et mimesis, le narrateur intervient très volontiers dans son récit, sollicite son destinataire, attire son regard, se tourne vers un public, tout comme dans la pièce Peter s'adresse aux spectateurs, leur demande d'affirmer leur croyance aux fées, de se manifester physiquement en applaudissant pour faire revivre Tinker Bell mourante. À de multiples reprises, la lecture se fait interactive. Soit l'adulte explique, précise, montre le chemin, soit il invite l'enfant à participer, à coopérer dans l'acte d'écriture, dans le geste dramatique, à se faire auteur, narrateur, acteur.

Barrie, l'un des écrivains les plus célèbres de son temps, est très ancré dans l'époque édouardienne. Peter Pan fait directement écho à ce monde littéraire et artistique. L'auteur attribue à ses personnages le nom de ses proches, à commencer bien entendu par ceux des fils Davies auxquels l'œuvre est adressée. Il attribue à Mr. Darling le prénom de l'aîné des Davies, George, lui-même ainsi nommé en hommage à son grand-père George du Maurier, ami d'Henry James ${ }^{4}$, dont le fils Gerald jouait à la fois le rôle de Hook et de Mr. Darling. L'aîné des fils Darling est John, prénom du deuxième fils Davies; le héros éponyme doit son prénom à Peter, troisième des Davies, qui lui-même doit le sien à Peter Ibbetson, roman de George du Maurier ; le dernier-né des Darling se prénomme Michael, comme le quatrième Davies. Le second prénom de John, Napoléon, est un hommage à Frohman, ami et producteur de Barrie, fervent admirateur de l'empereur qui apparaît à plusieurs autres reprises dans le texte, en particulier dans une scène majeure qui est la réplique du tableau de Sir William-Quiller Orchardson : Napoleon on Board HMS Bellerophon (1881), représentant l'exilé sur le navire en route pour Sainte-Hélène. L'un des pirates, Alf Mason, porte le nom d'un ami romancier et dramaturge, A.E.W. Mason; Cecco, pirate sur le «Jolly Roger ", a le prénom du fils d'un autre ami romancier, Maurice Hewlett, auteur en 1898 de Pan and the Young Sheperd. Le prénom Wendy, qui n'existait pas auparavant, est un hommage à la fille, morte prématurément, de son éditeur et ami écrivain, Henley ${ }^{5}$, lequel, éditeur aussi et collaborateur de Stevenson sur plusieurs pièces de théâtre, inspira à dernier le personnage de Long John Silver de Treasure Island. Quand à James Hook, il porte simplement le prénom de James Matthew Barrie auquel ce dernier déclare volontiers s'identifier. 
6 Nombreuses sont en outre les références à des œuvres littéraires. Peter Pan, dont l'île magique évoque celle de Prospero, rappelle à la fois Ariel et Puck, tandis que dès le premier chapitre de The Little White Bird qui lui est consacré, Barrie le place sous le signe du romantisme en introduisant le personnage de Shelley ${ }^{6}$, auteur de Queen Mab, reine des fées à laquelle il est fait allusion à plusieurs reprises :

Nevertheless, Peter did reach the Gardens at last by the help of Shelley's boat, as I am now to tell you.

Shelley was a young gentleman and as grown-up as he need ever expect to be. He was a poet; and they are never exactly grown-up. They are people who despise money except what you need for to-day, and he had all that and five pounds over. So, when he was walking in the Kensington Gardens, he made a paper boat of his bank-note, and sent it sailing on the Serpentine ${ }^{7}$.

7 Le jeune Peter navigue donc sur la Serpentine grâce à Shelley dont la première femme se suicida à l'âge de vingt-et-un ans en jetant dans cette même Serpentine et qui lui-même se noya à trente ans au large de La Spezia. Les mots de Hook « I'll show you now the road to dusty death ${ }^{8}$ » rappellent " the way to dusty death » qu'évoque Macbeth (acte V, scène 5). Barrie joue sans cesse sur l'intertextualité. Ainsi le titre du chapitre 3, invitation au voyage, "Come Away, come Away», reprend «The Forsaken Merman» (1849) de Matthew Arnold «Come, dear children, let us away " et «The Stolen Child» dans The Wanderings of Oisin (1889) de Yeats : "Come away, O human child! To the waters and the wild. With a faery, hand in hand [... $]^{10} »$ Barrie, lecteur passionné, nourri de la Bible, des grands auteurs, mais aussi de "penny dreadfuls ", de littérature populaire et de romans d'aventures, se tourne également vers Cooper, Marryat Haggard, Henty, Ballantyne, très lus par les jeunes garçons de l'époque. Ses Indiens doivent beaucoup à ceux de Fenimore Cooper, son île à The Coral Island (1857) de Ballantyne - que reprendra Golding en 1954 dans Lord of the Flies - pour lequel en 1913 il rédige une préface où il déclare « To be born is to be wrecked on an island ${ }^{11}$ ", reprenant ainsi l'un des thèmes majeurs de Peter Pan. Il pastiche volontiers The Swiss Family Robinson de Wyss - roman lui-même basé sur le Robinson Crusoe de Defoe - dont la première traduction en anglais (1816) est attribuée à Godwin, père de Mary Shelley ${ }^{12}$. L'effrayant mais pathétique Hook, ennemi mortel de Peter, dont la parenté est sombre et mystérieuse, qui est en quête d'une mère et finit précipité dans les flots, n'est d'ailleurs pas sans rappeler la créature de Frankenstein qui parcourt le monde à la recherche d'un père.

Dès qu'ils se font rares dans les Caraïbes, à la fin du XviII ${ }^{\mathrm{e}}$ siècle, les pirates envahissent la fiction. Pour Hook, Barrie s'inspire sans doute de fameux ouvrages sur la piraterie, dont $A$ General History of the Robberies and Murders of the Most Notorious Pyrates, du capitaine Charles Johnson, tardivement attribué à Defoe. Mais Hook rappelle aussi d'autres figures de forbans célèbres, devenues légendaires et personnages génériques du roman d'aventure: écumeurs des mers terrifiants, amputés, certains dandys, d'autres poètes, tous fascinants. L'auteur emprunte au capitaine Cook et à Bartholomew Roberts et mentionne William Kidd, Harry Morgan, Blackbeard et Teach - ce dernier nommé par Stevenson dans The Master of Ballantrae. De même que Wendy reconnaît Peter, figure de légende, bien avant leur rencontre, de même, à peine arrivés à Neverland, dès que les fils Darling entendent prononcer le nom de Hook, ils l'identifient aussitôt comme figure littéraire :

Then indeed Michael began to cry, and even John could speak in gulps only, for they knew Hook's reputation. 
He was Blackbeard's bo'sun," John whispered huskily. "He is the worst of them all.

$\mathrm{He}$ is the only man of whom Barbecue was afraid ${ }^{13}$.

Là encore d'autres histoires se tissent dans le récit. Barrie fait entrer et défiler ses pirates comme sur une scène, en accumulant les détails pittoresques « Here is Bill Jukes, every inch of him tatooed, the same Bill Jukes who got six dozen on the Walrus from Flint ${ }^{14}$. $\gg$ Le récit de Stevenson s'insère à plusieurs niveaux dans celui de Barrie : Flint, capitaine du Walrus est un personnage essentiel de Treasure Island, bien que déjà mort quand débute le récit de Stevenson. Seacook, autre forban de Peter Pan, est en fait le titre d'origine de Treasure Island lors de sa première publication dans le magazine Young Folks de 1881 à 1882. Et Hook est censé avoir naguère vaincu Barbecue, surnom de Long John Silver de Stevenson. Ainsi, Barrie rend hommage à son maître en croisant ses propres personnages avec les siens, qui s'inscrivent ainsi en toile de fond du pays imaginaire. Et le lecteur adulte familier du texte source, sensible à l'intertextualité, retrouve un horizon littéraire familier et établit une forme de connivence ludique avec l'auteur.

Neverland, espace foisonnant, hétérogène, saturé, offre une image différente à chacun. Dès leur arrivée, alors que l'île magique devient réelle à leurs yeux, les enfants reconnaissent sans hésiter leur monde imaginaire, comme un rêve soudain réalisé : Michael retrouve sa grotte, John son bateau et son flamant, Wendy son louveteau abandonné. Ce pays de jamais où l'on revient toujours, prend forme de collage où voisinent de façon cocasse toutes sortes de souvenirs d'enfance de l'auteur, et ses lectures volontiers pastichées. S'adressant au jeune lecteur, le narrateur dessine la carte qui précède bien des récits d'aventures en accumulant pêle-mêle, sous forme de liste incongrue, une série d'images hétéroclites du monde de l'enfance :

I don't know whether you have ever seen a map of a person's mind. Doctors sometimes draw maps of other parts of you [...] but catch them trying to draw a map of a child's mind, which is not only confused, but keeps going round all the time. There are zigzag lines on it, just like your temperature on a card, and these are probably roads in the island; for the Neverland is always more or less an island, with astonishing splashes of colour here and there, and coral reefs and rakishlooking craft in the offing, and savages and lonely lairs, and gnomes who are mostly tailors, and caves through which a river runs, and princes with six elder brothers, and a hut fast going to decay, and a very small old lady with a hooked nose [...] there is also first day at school religion, fathers, the round pond, needlework, murders, hangings, verbs that take the dative, chocolate-pudding day; getting into braces, say ninety-nine, threepence for pulling out your tooth yourself, and so on. “ [...] Of course the Neverlands vary a great deal. John's for instance, had a lagoon with flamingoes flying over it at which John was shooting, while Michael, who was very small, had a flamingo with lagoons flying over it. [...] Wendy had a pet wolf forsaken by its parents $[. . .]^{15}$.

11 Puis, quelques lignes plus loin, changeant soudain de ton, s'adressant par-dessus l'épaule de l'enfant à l'adulte aux côtés duquel il se situe, Barrie conclut sur le ton de la nostalgie :

On these magic shores children at play are for ever beaching their coracles. We too have been there; we can still hear the sound of the surf, though we shall land no more ${ }^{16}$.

La position du narrateur devient incertaine : est-il à Neverland ou dans le monde réel, participant à l'histoire ou en mesure de la raconter précisément parce qu'il se situe à l'extérieur? Barrie, qui se disait si proche de Peter Pan, qui exprimait volontiers sa difficulté, sa douleur, de devoir renoncer aux jeux et au plaisir de l'enfance, annonce cette fatalité et déclare, dès les premières lignes du roman, comme on énonce un postulat: 
"All children, except one, grow up ${ }^{17}$. " Suit aussitôt une courte scène où Mrs Darling, voyant la petite Wendy, âgée de deux ans, lui apporter une fleur fraichement cueillie, s'écrie : "Oh, why can't you remain like this for ever ». This was all that passed between them on the subject, but henceforth Wendy knew that she must grow up. You always know after you are two. Two is the beginning of the end ${ }^{18}$. "

13 Ainsi, c'est entre mères et filles que se dit, implicitement, l'avenir. Au chapitre suivant, Mrs. Darling surprend ses propres enfants " playing at being herself and father on the occasion of Wendy's birth [... $]^{19}$. » La mère devient spectatrice de sa maternité mise en scène par sa propre progéniture. Là encore, comme dans The Little White Bird, la procréation est douloureusement en jeu, la naissance en question : " Then John was born, with the extra pomp that he conceived due to the birth of a male, and Michael came from his bath to ask to be born also, but John said brutally that they did not want any more ${ }^{20}$."

Ces fréquentes mises en abyme, ces récits enchâssés, eux-mêmes souvent enchâssants, ces décalages dans les temps de la narration font écho à une image récurrente chez Barrie : celles de boîtes gigognes rappelant cassettes et coffrets, coffres au trésor du roman d'aventures aussi. Dès le premier chapitre, Mrs. Darling est présentée en ces termes :

Her romantic mind was like the tiny boxes, one within the other, that come from the puzzling East, however many you discover there is always one more. $\mathrm{He}-\mathrm{Mr}$ Darling - got all of her, except the innermost box and the kiss ${ }^{21}$.

15 Avant même de surprendre dans son sommeil Mrs. Darling, puis bientôt sa fille Wendy, et une génération plus tard la fille de celle-ci, Peter Pan est un personnage familier des enfants. Wendy, imaginant que la nuit il vient jouer de la flûte pour elle, ravive les souvenirs de sa mère :

"But who is he, my pet?"

"He is Peter Pan, you know, mother."

At first Mrs Darling did not know, but after thinking back into her childhood she just remembered a Peter Pan who was said to live with the fairies. There were odd stories about him; as that when children died he went part of the way with them, so that they should not be frightened ${ }^{22}$.

16 L'enfant-fée est donc aussi une figure de Charon, un nocher des enfers juvénile et affectueux. Alors que Mr. Darling ne voit là que fadaises et balivernes, Mrs Darling reconnaît Peter comme personnage de récit, alors que lui-même la connaît comme conteuse. Ayant entendu Mrs. Darling raconter à ses enfants "Cendrillon », Peter invite Wendy à Neverland pour reprendre le récit « About the prince who couldn't find the lady who wore the glass slipper ${ }^{23}$ ", l'auteur réintroduisant une nouvelle fois une histoire dans l'histoire. Étonnée par l'erreur de Peter quant à l'interprétation de Cendrillon, Wendy, qui ne perd jamais de vue épousailles et progéniture, rectifie: "Peter", said Wendy excitedly, "that was Cinderella, and he found her, and they lived happy ever after ${ }^{24}$." Histoire très attendue et bientôt relayée par l'un des garçons perdus, Slightly, qui déclare «I wish he - Peter - would come back, and tell us whether he has heard anything more about Cinderella. They talked of Cinderella, and Tootles was confident that his mother "must have been very like her ${ }^{25}$. " Ainsi souvenir du réel et fiction se répondent. Et à nouveau la littérature parle de littérature. Le retour de Peter signifie reprises de récits en même temps que reprise d'aventures - elles-mêmes inspirées d'une multiplicité de textes littéraires - qui deviendront récits à leur tour puisqu'au fil des générations les petites filles devenues mères se feront narratrices. En outre, au chapitre 11 - en abyme et sous forme d'analepse lui aussi - intitulé "Wendy's Story" la petite fille, craignant qu'à Neverland ses frères n'oublient le monde réel - ce qui peu à peu est le cas - leur raconte 
leur propre histoire, leur existence passée au sein de leur famille londonienne, reprenant, parodiant presque, une scène de conte traditionnel à la veillée :

"Listen, then",said Wendy, settling down to her story, with Michael at her feet and seven boys in the bed. "There was once a gentleman -"

"I had rather he had been a lady", Curly said.

"I wish he had been a white rat", said Nibs.

"Quiet, their mother admonished them. "There was a lady also, and -26"

17 Alors que cette scène se prolonge, les garçons perdus interagissent constamment avec la narratrice, qui, outre son rôle de véritable sœur, joue celui de mère de substitution. Lorsqu'elle en arrive au récit de la rencontre entre ses frères et les garçons perdus, alors que se croisent imaginaire et réel, Tootles, radieux, s'écrie : « I am in a story. Hurray, I am in a story, Nibs ${ }^{27}$. » Là encore le texte s'inscrit clairement dans la métafiction.

Dans Peter Pan, tous les personnages principaux donnent à voir une identité fragmentée, démultipliée. Ainsi Mrs. Darling, présentée dès les premières lignes comme «a lovely lady, with a romantic $\operatorname{mind}^{28}$ ", qui joue les femmes soumises et les mères parfaites, est aussi une épouse dominatrice, qui sous couvert de tendresse rend son mari dangereusement immature et une mère abusive qui furète dans les pensées de ses enfants et les empêche de grandir. Portant sur elle un regard étranger et ironique, Barrie, passant sans cesse du statut d'auteur à celui de narrateur, prend à témoin le narrataire, lui confie son indécision quant au jugement à porter sur le personnage, comme si celui-ci lui échappait : "You see, the woman had no proper spirit. I had meant to say extraordinary nice things about her ; but I despise her, and not one of them will say now ${ }^{29}$. »

Puis, se situant à l'extérieur de son texte, comme spectateur parmi d'autres, renouant ainsi avec le genre théatral, il déclare : « Now that we look at her closely [...] I won't be able to say nasty things about her after all ${ }^{30}$. »

Mr Darling, banquier impécunieux, époux médiocre et père raté, veule et hypocrite, exige des siens - notamment de sa femme qu'il ne cesse d'appeler « Maman » - obéissance voire admiration, tout en se comportant comme un gamin irresponsable. À la naissance de chacun de ses enfants il échafaude froidement des projets infanticides au nom du simple bon sens. Puis, se sentant à juste titre coupable de leur fuite, il élit domicile dans la niche de la chienne qui remplissait - fort bien au demeurant - le rôle de nurse. Remportant quelque succès en se rendant au bureau dans cet équipage, il s'exhibe bientôt dans tout Londres - où les zoos humains étaient alors très prisés - non sans en tirer une certaine forme de plaisir. Comédien ambulant et Don Quichotte à la fois, caricature pathétique de l'acteur et du metteur en scène, il acquiert à sa manière une dimension dramatique et une authentique grandeur. À la scène que jouent les enfants Darling dans le rôle de leurs propres parents au moment de leur naissance, répondent les scènes où leur père s'affiche dans le rôle de la chienne. Barrie porte ici un regard amusé sur le monde du théâtre qu'il connaît si bien, sur les acteurs, les metteurs en scène, le public et la critique :

Inwardly he must have suffered torture; but he preserved a calm exterior even when the young criticized his little home, and he always lifted his hat courteously to any lady who looked inside.

It may have been quixotic, but it was magnificent. Soon the inward meaning of it leaked out, and the great heart of the public was touched. Crowds followed the cab, cheering it lustily; charming girls scaled it to get his autograph; interviews appeared in the better class of papers, and society invited him to dinner and added, "Do come in the kennel ${ }^{31}$." 
21 etit bourgeois étriqué en mal de respectabilité se réalise finalement en s'exposan comme une bête de foire ; le récit domestique bascule alors dans le spectacle burlesque. De la multiplication et du transfert incongru des rôles naît un regard critique sur la société. Si le rôle social imparti, imposé, ne convient pas à l'individu, pourquoi ne pas en choisir un autre, tout différent, certes inattendu voire ridicule, mais somme toute plus gratifiant?

Mais c'est avec Peter et Hook que les identités multiples, les glissements de rôles sont les plus spectaculaires. Le nom même de Peter Pan affiche sa double identité. Peter est le prénom, on l'a vu, du troisième fils Davies tandis que Pan fait référence au dieu grec qui a beaucoup en commun avec le héros de Barrie. Joueur de flûte et en perpétuel mouvement lui aussi, Pan, le plus jeune des dieux, qui sème autour de lui la panique, est rejeté par sa mère dès sa naissance, et transporté sur l'olympe par son père, qui ne le reconnaît pas comme fils. Pan est très présent dans la littérature victorienne et édouardienne. Virginibus Puerisque and other Papers de Stevenson (1881) comprend un essai intitulé «Pan's Pipes», Arthur Machen publie dix ans plus tard The Great God Pan, Kenneth Grahame place le chapitre intitulé « The Pipers at the Gates of Dawn » au coeur même de The Wind in the Willows (1908) dont le frontispice des premières éditions, de Walford Graham Robertson, représente le dieu Pan.

En outre, Peter Pan, comme le souligne Anne Yeoman est à la fois Icare, Prométhée et Lucifer $^{32}$, tandis que Nathalie Prince voit en lui une figure caïnique ${ }^{33}$. Peter Pan, qui joue une multitude de rôles, se plaît à en changer brutalement, adoptant soudain celui d'un ennemi, et impose à sa guise pareils changements à son entourage souvent désemparé. Les métamorphoses se font souvent anamorphoses. Peter imite si fidèlement le tic-tac du crocodile que tous s'y méprennent, lui-même y compris. Lorsqu'il prétend être Hook, ce dernier s'y trompe lui aussi et, abasourdi, se questionnant sur sa propre identité, il s'écrie: "If you are Hook,» he said almost humbly, «come, tell me, who am $\mathrm{I}^{34}$ ?» Échappant à toute catégorie, ami du soleil et des étoiles, Peter est pan, «le tout ». Face à Peter, le puer insolent, toujours virevoltant, image de l'enfance absolue, arrogante et jubilatoire, se dresse Hook le senex amputé. Écumeur des mers, sur son bateau à l'ancre, il est profondément ancré dans le réel, dans l'histoire, dans la culture, la légende et la littérature. Alors que le physique de Peter, qui refuse que quiconque le touche, est à peine évoqué, Hook est décrit avec précision : basané, squelettique, longues mèches brunes, regard bleu - pervenche ou myosotis, c'est selon - ou enflammé lorsqu'il se prend de colère, il est élégamment vêtu et méprise les sots et les brutes de son équipage qu'il traite de chiens. Alors que Peter ne sait ni lire ni écrire, Hook est cultivé, amateur de poésie, joueur de clavecin à ses heures, "raconteur ${ }^{35}$ of repute»; il parle volontiers une langue archaïsante, avec une diction parfaite, et fume non pas la pipe du matelot mais le cigare de l'aristocrate. Ancien du prestigieux collège d'Eton où grâce à Barrie étudièrent les fils Davies, Hook apparaît comme la parodie du héros romantique. Rêveur, il exhale de profonds soupirs devant la beauté sereine du soir avant de raconter avec mélancolie à son bosco l'histoire de sa vie, dont Peter fait partie intégrante. La gentille petite Wendy n'est pas insensible à son charme. Lorsqu'il lui donne le bras pour monter sur son bateau crasseux - crasse que pourtant elle remarque et déplore - la demoiselle se sent si troublée que le roman d'aventures manque un instant de virer au roman d'amour. Hook est terrifié par la mort qui le poursuit sous la forme du crocodile dévoreur entre les mâchoires duquel Peter a jadis jeté sa main droite. Contrairement à celui-ci, qui n'a d'autres souvenirs que sa fuite initiale, Hook semble lié aux Stuart maudits, ces souverains au 
destin tragique auxquels physiquement il ressemble et qu'il se plaît à singer dans sa mise. Le capitaine des pirates paraît cacher un mystère, un secret d'état peut-être qui, révélé, mettrait le royaume en péril. On peut en effet s'interroger sur l'identité de Hook avant le geste de Peter et la pose du fameux crochet de fer dont il tire tant de fierté, et qu'en fait il doit, de même que son nom, à son ennemi. Pirate sanguinaire, il exécute illico sans remords, d'un revers de son crochet celui qui, l'ayant bousculé par mégarde, a risqué d'abîmer son col en dentelle. Passant là encore du récit au jeu, le narrateur propose d'y participer. Comme si, pour lui de même que pour les protagonistes, jouer à tuer était la plus délicieuse des distractions. «Let us now kill a pirate, to show Hook's method ${ }^{36}$ » et, tenant le narrataire en haleine, anticipant la suite il ajoute: "Such is the terrible man against whom Peter Pan is pitted. Which will win ${ }^{37}$ ?» Hook, qui se désole de ne pas être aimé des petits enfants, tout comme Peter demande à Wendy d'être sa mère «Ah, envy not $\mathrm{Hook}^{38}$ » conseille le narrateur et, plus loin, adoptant le ton du chroniqueur et livrant son récit au jugement du spécialiste, il déclare, ouvrant sur de nouveaux textes à venir : " To what extent Hook is to blame for his tactics on this occasion is for the historian to decide ${ }^{39}$. " Après la défaite de Hook, il rend hommage à celui-ci, et saluant sa sortie de scène, il intervient encore: «Misguided man though he was, we may be glad, without sympathizing with him, that in the end he was true to the traditions of his race [...] James Hook, though not wholly unheroic figure, farewell ${ }^{40}$. »

Lorsque Peter et Hook s'affrontent en combat singulier sur le rocher, visage contre visage, c'est bien le double, le doppelgänger - étymologiquement à la fois «sosie» et " compagnon de route " - le reflet l'un de l'autre, tous deux reflets de l'auteur aussi, qui se donne à voir ; ils croisent le fer avec la même efficacité et le même brio. Rebelles, en quête d'amour maternel, ils ont refusé l'ordre social; exilés volontaires ils se sont mis hors la loi, ne reconnaissant d'autre règle que celle qu'ils imposent. À la moindre marque d'irrespect de la part de leurs compagnons, ils tuent. Entre celui qui refuse de grandir et celui qui a peur de mourir il n'y a qu'un pas. Hook apparaît à Peter comme une image de lui-même devenu adulte, ce qu'il ne peut supporter. À l'inverse Hook voit en lui l'insolente jeunesse qu'il a perdue alors que la mort le talonne. Tous deux capitaines, l'enfant radieux, l'enfant-fée qui relève du merveilleux, et l'homme sombre, le vieux forban du roman d'aventures, doubles antagonistes, se livrent, depuis un temps antérieur au récit, une lutte à mort. Lorsque Hook se voit vaincu, il demande à Peter de lui épargner le coup de lame fatal et de l'aider à disparaître en le faisant basculer dans les flots d'un coup de pied, ce pied auquel Wendy avait naguère recousu l'ombre perdue. L'avantdernier chapitre « Hook or me this time » - que l'on peut lire "Hook is ${ }^{41}$ me this time », se clôt sur la métamorphose du capitaine Pan en capitaine Hook. Après sa victoire sur les pirates et l'exécution de quinze d'entre eux, Peter, redevenu petit garçon perdu, passe la nuit à sangloter dans les bras de Wendy. Puis, dès le lendemain matin, il se met en scène sur le pont du navire pirate et reprend fidèlement le rôle de son ennemi disparu. Faisant fusionner le modèle et la réplique, il devient le double de son double en somme, brandissant à son tour son crochet « like a hook » :

It need not be said who was the captain. [...] Some of them wanted it to be an honest ship and others were in favour of keeping it a pirate; but the captain treated them as dogs, and they dared not express their wishes to him even in a round robin. Instant obedience was the only safe thing. Slightly got a dozen for looking perplexed when told to take soundings. The general feeling was that Peter was honest just now to lull Wendy's suspicions, but that there might be a change when the new suit was ready, which, against her will, she was making for him out of some of Hook's wickedest garments. It was afterwards whispered among them that on the 
first night he wore this suit he sat long in the cabin with Hook's cigar-holder in his mouth and one hand clenched, all but for the forefinger, which he bent and held threateningly aloft like a hook ${ }^{42}$. littéraire et demeure dans le sillage de Stevenson : l'auteur de Treasure Island (1883) et de The Master of Ballantrae (1889) est aussi celui de The Strange Case of Dr. Jekyll and Mr. Hyde (1885). Le motif du double, du double antagoniste en particulier, présent dans les textes depuis les civilisations les plus anciennes où sosies et frères jumeaux ennemis sont pléthore, est développé en Grande-Bretagne notamment par Godwin dans The Adventures of Caleb William (1794) et par Lewis dans The Monk (1796). On le retrouve chez Maturin dans Melmoth the Wanderer (1820) puis chez Wilde dans The Picture of Dorian Gray en 1891. Le double - ainsi que l'ombre perdue, vendue ou volée - développé par Hoffman dans plusieurs textes, fut un thème de prédilection des Romantiques allemands. Chamisso, qui influença tant Hoffman, était sans doute connu de Barrie, L'Histoire merveilleuse de Peter Schlemihl ou l'homme qui a vendu son ombre (1814) étant alors très lu outre-Manche, même par les plus jeunes. Barrie reprend le thème de l'ombre absente. C'est pour retrouver celle-ci, malencontreusement arrachée, que Peter revient chez les Darling; retour essentiel puisqu'il marque la rencontre avec Wendy et le départ vers Neverland. En recousant cette ombre au pied du jeune garçon, Wendy redonne au personnage sa substance, mais aussi sa capacité de force procréatrice - en vain puisque l'idée d'engendrer, et même d'être engendré, lui fait horreur ${ }^{43}$.

Pan fonctionne en outre sur une série de duplications: scènes redoublées, phrases répétées, anecdotes et détails repris, jumeaux comme ces garçons perdus dont le nom n'est pas mentionné, qui, toujours confondus, disent la difficulté d'être doubles. Hook est fier à la fois de son double porte-cigares et de son crochet, à l'origine double lui aussi. Deux combats opposent les deux capitaines, deux rencontres ont lieu entre lui et la mèreoiseau. Après son départ initial de sa propre nursery, Peter y revient à deux reprises, de même qu'il s'introduit deux fois chez les Darling. Deux interprétations d'une même situation sont parfois proposées. Ainsi, lorsque Peter explique à Wendy que sa mère l'a oublié, le narrateur ajoute : «I am not sure that this was true, but Peter thought it was true ${ }^{44}$. » Deux scènes en miroir représentent son arrivée dans la même nursery, à une génération d'intervalle. Et c'est exactement dans les mêmes termes que Wendy et sa fille Jane, si semblables, réveillées l'une et l'autre par Peter, l'accueillent : «Boy, why are you crying ${ }^{45}$ ?» Le texte a même une double fin avec "When Wendy Grew up: An Afterthought ", publié à titre posthume et désormais joint aux éditions de la pièce et du roman. «La réalité humaine semble ne pouvoir commencer qu'avec la seconde fois. Une mesure pour rien [...] » écrit Clément Rosset ${ }^{46}$. Dans le récit de Barrie, c'est la seconde fois qui est décisive, en dépit de l'affirmation de Solomon Caw dans The Little White Bird: "There is no second chance, not for most of us ${ }^{47}$."

La prolepse finale fait écho aux nombreuses analepses du récit et ouvre sur l'infini. Le passé de la narration fait place au futur. Dans les dernières pages du roman, le narrateur évoque la disparition des membres de la famille Darling, mentionnant leur mort passée ou prochaine sans la moindre émotion, comme s'il se débarrassait d'une histoire en la

Belphégor, 10-3 | 2013 
reléguant dans un passé lointain : «Mrs Darling was now dead and forgotten. [...] Nana also had passed away. She died of old age, and at the end she had been rather difficult to go on with ${ }^{48}$.» Et il reprend une formule consacrée du conte traditionnel «[...] all this happened long ago $^{49}$.»

En même temps, il ébauche la liste des descendantes de Wendy. Ces petites filles en série, se feront d'abord personnages de nouvelles aventures puis narratrices de l'histoire de Peter, toujours plus riche à mesure des générations. Et ainsi se multiplieront les récits tant que les enfants seront «gay and innocent and heartless ${ }^{50}$.» Ces mots, significatifs à une époque qui a vu naître la psychanalyse, sont répétés comme un leitmotiv non moins de cinq fois à la fin du texte que finalement elle clôture : "When Margaret grows up she will have a daughter, who is to be Peter's mother in turn ; and thus it will go on, so long as children are gay and innocent and heartless ${ }^{51}$. »

Les personnages et l'histoire de Peter Pan qui prolifèrent de diverses manières depuis leurs multiples origines, si bien qu'ils semblent échapper à leur auteur relèvent, du point de vue thématique et narratologique, de la prolifération et du débordement.

À Londres, les Darling, sous couvert de respect des règles et de mesure, affichent l'extravagance. Peter Pan lui-même, échappant à l'encadrement de la fenêtre ouverte/ fermée, en perpétuel mouvement, règne sur un espace, ou plutôt un décor, exotique foisonnant et saturé où se mêlent une multitude de genres et des personnages hétérogènes. Dans le récit lui-même, où mises en abyme et récits enchâssés abondent, où se brouillent les figures de l'auteur, tout est profusion. Ainsi Peter Pan, la pantomime certes, mais aussi le roman, paraît relever de l'esthétique baroque. Se déployant tel un pliage immense, multiforme, extrêmement mobile, jouant sur la métamorphose et l'illusion, l'œuvre de Barrie est comme un "pli qui va à l'infini » selon l'expression de Deleuze $^{52}$. Sous le signe de la multiplication et du dérèglement, toujours elle se dédouble et recommence. Et tant que les enfants seront "gay, and innocent and heartless ${ }^{53}$ " l'histoire de Peter Pan recommencera.

\section{BIBLIOGRAPHIE}

\section{Euvres de Barrie}

The Little White Bird (1902), Fairfield, 1st World Library, 2006.

Peter Pan (le roman) (1911), Londres, Puffin Classics, 1994.

A Kiss for Cinderella (1916), Londres, Hodder and Stoughton, 1920.

Peter Pan or the Boy Who Would Not Grow up (1928), in Peter Pan and other Plays, Oxford, Oxford University Press, The World's Classics, 1995.

Better Dead (1888), Charleston, BiblioBazaar, 2008.

The Adventure of the Two Collaborators (1893), London, Roger Lancelyn Green, 1982.

Ouvrages critiques sur Barrie et sur Peter Pan 
Andrew Birkin, J.M. Barrie and the Lost Boys, New Haven and London, Yale University Press, 2003. Monique Chassagnol, Nathalie Prince, Isabelle Cani, Peter Pan, figure mythique, Paris, Autrement, 2010.

Roger Lancelyn Green, Fifty Years of Peter Pan, Londres, Peter Davies, 1954.

Jacqueline Rose, The Case of Peter Pan, or the Impossibility of Children's Fiction, Londres, The Macmillan Press, 1994.

Anne Yeoman, Now or Neverland, Peter Pan and the Myth of Eternal Youth, Toronto, Inner City Books, 1998.

Autres

Matthew Arnold, « The Forsaken Merman », dans The Strayed Reveller, and other Poems, 1849.

Dave Barry/Ridley Pearson, Peter and the Starcatchers, New York, Disney Ed. Hyperion, 2004.

Charles Baudelaire, «Les fenêtres » Petits Poèmes en prose/Le Spleen de Paris (1855-1864), Paris, Gallimard Folio, 2010.

Lewis Carroll, Through the Looking-Glass and What Alice Found There (1872), in The Annotated Alice, Londres, Penguin, 1970, p. 266.

Fabrice Colin, CyberPan, Paris, Mango Jeunesse, 2003.

Richard Comballot (Éd.), Les ombres de Peter Pan, Paris, Mnemos, 2004.

Arthur Conan Doyle, Memories and Adventures (1924), Londres, Wordsworth Classics, 2007.

Gilles Deleuze, Le pli : Leibniz et le baroque, Paris, Éditions de Minuit, 1988.

Clotilde Escale, La vieillesse de Peter Pan, Paris, Cherche Midi, 2006.

J.V. Hart, Capt. Hook, The Adventures of a Notorious Youth, New York, Laura Geringer Books/ HarperCollins, 2005.

Gunn Jeannie, We of the Never Never (1908), Charleston, BiblioBazaar, 2008.

Henry Lawson, « The Never Never Country » in When I was King and other Verses (1901) Sydney, Angus and Robertson, 1906.

David Lodge, Author, Author, Londres, Random House, 2004.

Régis Loisel, Peter Pan, Rennes, Vent d'Ouest : - Londres, 1990 - Opikanoba, 1992 - Tempête, 1994 Mains Rouges, 1996 - Crochet, 2002 - Destins, 2004.

Geraldine McGaughrean, Peter Pan in Scarlet, Oxford, Oxford University Press, 2006.

Alfred de Musset « La nuit de décembre » (1835)dans Poésies Complètes, Paris, Livre de Poche, Les Classiques de Poche, 2006.

François Rivière et Françoise Balibar, Les ailes de Peter Pan, Paris, Seuil, 1999.

Clément Rosset, Le réel et son double, Paris, Gallimard, 1976.

Percy Bysshe Shelley, Queen Mab (1813), The Poetical Works of Shelley, Cambridge, Cambridge University Press, 1975.

R.L. Stevenson, «The Suicide Club », dans New Arabian Nights (1882), Toronto, Dover Thrift Edition, 2000.

W.B. Yeats, «The Stolen Child» dans The Wanderings of Oisin, Paris, Verdier, éd. bilingue 2003 


\section{NOTES}

1. Le temps du passage de l'été à l'hiver est aussi celui du poème de Musset «La nuit de décembre" (1835) où l'étranger vêtu de noir, le frère, le solitaire, poursuit le poète depuis l'enfance.

2. Charles Baudelaire, "Les fenêtres" Petits Poèmes en prose /Le Spleen de Paris, Paris, 1854, Gallimard Folio, 2010, p. 119.

3. J. M. Barrie, Peter Pan (le roman), op.cit., Londres, Penguin, Puffin, 1994, p. 111 à 113.

4. Dans Author, Author (2004), David Lodge évoque l'amitié entre les deux hommes.

5. L'enfant, très attachée à Barrie, l'appelait «my friendy» mais, trop petite pour parler correctement, prononçait « my fwendy », « my wendy ».

6. Cette allusion, d'emblée, à Shelley, permet de mesurer l'importance des références littéraires chez Barrie tout en situant approximativement la naissance de Peter. Si l'on considère que Shelley commença à écrire vers 1810 et publia en 1813 Queen Mab que Barrie mentionne à plusieurs reprises, on peut dater la naissance de Peter vers cette époque.

7. J.M. Barrie, The Little White Bird, op.cit., p. 110-111.

8. Peter Pan and other Plays, op.cit., p. 146.

9. Matthew Arnold, « The Forsaken Merman », dans The Strange Reveller and other Poems, 1849.

10. W.B. Yeats, «The Stolen Child» dans The Wanderings of Oisin, Paris, Verdier, 2003, p. 134-137.

11. Cité par Naomi Lewis dans l'introduction de Peter Pan (le roman), op.cit., p. 6.

12. Barrie a le goût du pastiche et en écrit plusieurs dont Better Dead (1888), parodie de The Suicide Club de Stevenson, et The Adventure of the Two Collaborators (1893) en souvenir d'une pièce qui connut fort peu de succès, écrite avec Conan Doyle, son compatriote écossais, autre ami des fées. Ce dernier publie ce texte pour la première fois en 1924 dans son autobiographie intitulée Memories and Adventures.

13. Peter Pan (le roman), op.cit., p. 64.

14. Ibid., p. 75.

15. Ibid., p. 13-14.

16. Ibid. p. 14.

17. Ibid., p. 7.

18. Lewis Carroll accorde un peu plus de temps à l'enfance puisque Humpty Dumpty conseille à Alice « Leave off at seven ", Through the Looking-Glass, dans The Annotated Alice, Londres, Penguin, 1970.

19. Peter Pan (le roman), op.cit., p. 24.

20. Ibid.

21. Ibid., p. 7-8.

22. Ibid., p. 15.

23. Ibid., p. 47.

24. L'œuvre de Barrie résonne des échos faits à "Cendrillon", qu'il revisite dans A Kiss for Cinderella, comédie représentée pour la première fois à Londres en 1916. On y reconnaît plusieurs thèmes abordés dans Peter Pan. Durant la première guerre mondiale, une jeune fille pauvre qui prend en charge des enfants démunis s'imagine dans le rôle de Cendrillon. Ayant rencontré la fée marraine en la personne d'une infirmière de la Croix Rouge, elle finit en effet par trouver un prince Charmant inattendu dans le personnage attachant d'un agent de police local.

25. Peter Pan (le roman), op.cit., p. 80.

26. Ibid., p. 149.

27. Ibid., p. 150.

28. Ibid., p. 7. 
29. Ibid., p. 216.

30. Ibid., p. 218-219.

31. Ibid., p. 216-217.

32. Anne Yeoman, Now or Neverland, Peter Pan and the Myth of Eternal Youth, Toronto, Inner City Books, 1998, p. 31 à 68.

33. Monique Chassagnol et al., Peter Pan, figure mythique, Paris, Autrement, 2010, p. 95 à 100.

34. Peter Pan (le roman), op.cit., p. 124.

35. Peter Pan (le roman), op.cit., p. 76. « raconteur » est en français dans le texte. Pareil talent est un autre point commun entre l'auteur et son personnage.

36. Ibid., p. 77.

37. Ibid.

38. Ibid., p. 186.

39. Ibid., p. 164.

40. Ibid., p. 208.

41. C'est moi qui souligne.

42. Peter Pan (le roman), op.cit., p. 212-213.

43. Dans Die Frau ohne Schatten, (La Femme sans ombre),(1919), opéra de Strauss sur un livret de von Hofmannstahl, qui relève du Märchen allemand, l'héroïne, la fille du prince des esprits, ne peut donner d'enfants à son époux l'empereur tant qu'elle est dépourvue d'ombre.

44. Peter Pan (le roman), op.cit., p. 153.

45. Ibid., p. 37 et 240 .

46. Clément Rosset, Le réel et son double, Paris, Gallimard, 1976, p. 62.

47. J. M. Barrie, The Little White Bird, op.cit., p. 130. Par exemple, dans « Le Petit Poucet ", c'est le second abandon des parents qui entraîne la rencontre avec l'ogre, laquelle manque d'être fatale mais finalement fait la fortune du personnage éponyme et de sa famille. Dans «Cendrillon », la première soirée au bal est sans conséquence puisque la jeune fille, obéissant aux recommandations de la fée, rentre avant minuit. C'est lors de second bal qu'elle perd la fameuse pantoufle, et, après l'avoir essayée devant le prince, c'est en sortant de sa poche la seconde qu'elle justifie son identité et devient de ce fait princesse.

48. Peter Pan (le roman), op.cit., pp. 234- 235.

49. Ibid., p. 242.

50. Ibid., p. 236.

51. Ibid., 4 fois p. 236.1 fois p. 242.

52. Gilles Deleuze, Le Pli. Leibniz et le baroque, Paris, Éditions de Minuit, 1988, p. 5.

53. Voir note 64.

\section{AUTEUR}

\section{MONIQUE CHASSAGNOL}

Professeur émérite Université Paris-Ouest Nanterre 